\title{
Influence of Sparshanam, a medical humanities module on personal and professional development
}

\author{
Shankar, P.R. ${ }^{1}$, Dhakal, A.K. ${ }^{2}$, Piryani, R.M. ${ }^{1}$, Shrestha, D. ${ }^{2}$, Dwa, Y.P. \\ ${ }^{1}$ International Medical University, Malaysia, ${ }^{2}$ KIST Medical College, Lalitpur, Nepal, ${ }^{3}$ Bilawal Medical \\ College, Sindh, Pakistan
}

\section{Background:}

Medical humanities (MH) has several uses in medical education and $\mathrm{MH}$ programs are common in developed nations. However, in Nepal, a developing country in South Asia, these programs are still not common. A module was conducted for consecutive batches of first-year undergraduate medical students at a medical college in Nepal from 2009 to 2012. Studying the medium to the long-term impact of $\mathrm{MH}$ has many challenges. The present study aims to obtain the perception of the first batch of MBBS graduates about Sparshanam, the MH module conducted at the college, and its perceived impact on their personal and professional life.

\section{Methods:}

Responses were collected using an online form after obtaining written, informed consent online. Participants' perceptions about the strengths and weaknesses of the module, their current work, and the contribution of the module to their personal and professional development were studied. Information about gender, specialization, place, and country of work, and marital status was noted.

\section{Results:}

Thirty-nine of the 75 alumni (52\%) participated. Most were working in Nepal within the Kathmandu valley. Teamwork, interactive group sessions, and providing a holistic perspective about the patients were the strengths and offering of module during the clinical years and postgraduate training were recommended. The module enhanced respondents' teamwork, communication, and leadership skills, ability to empathize with patients, and clinical observation skills. They became better patient educators and counsellors. They wanted all medical colleges in Nepal to offer an $\mathrm{MH}$ module and for the discipline to be offered throughout the medical training including postgraduation. They were also of the opinion that the module developed their moral values and adaptability to different situations.

\section{Conclusion:}

The results from the evaluation of this module by working doctors may serve as a facilitating factor to introduce MH in Nepalese medical schools and elsewhere in the region.

Keywords: Medical Humanities, Moral Values 